\title{
A case of progressive encephalomyelitis with rigidity and positive antiglutamic acid dehydrogenase antibodies
}

\author{
David J Burn, Joanna Ball, Andrew J Lees, Peter O Behan, John A Morgan-Hughes
}

\begin{abstract}
A 50 year old woman developed progressive encephalomyelitis with rigidity over a three year period. Her CSF contained oligoclonal bands and both her serum and CSF contained antibodies directed against GABA-ergic synapses (antiglutamic acid dehydrogenase antibodies). These antibodies have recently been described in cases of stiff man syndrome. Both disorders may be part of a clinical spectrum that has an underlying autoimmune basis.
\end{abstract}

Progressive encephalomyelitis with rigidity (PEWR) is a rare disorder of unknown aetiology, characterised by muscular rigidity, abnormal postures, painful muscle spasms, and myoclonus. ${ }^{1}$ Recent reports of antiglutamic acid dehydrogenase (anti-GAD) antibodies in cases of stiff man syndrome have suggested an autoimmune basis for this condition, ${ }^{2}$ which bears some similarity clinically to PEWR. We report a case of PEWR with positive anti-GAD antibodies in both serum and cerebrospinal fluid (CSF). As far as we are aware, this is the first time this association has been reported and raises the possibility that PEWR may also have an autoimmune pathogenesis.

\section{Case report}

A 50 year old woman first developed symptoms in 1986, when she noticed weakness in her upper limbs when hanging washing or cleaning windows. There was no history of recent trauma or surgery. The muscles were not painful or tender. She wore a collar for three months without improvement and soon afterwards noticed stiffness and loss of range of movement in her neck. In addition, her head was occasionally pulled back in episodes lasting up to one hour. She was prescribed oral diazepam which eased the spasms. Since March 1987 she has had progressive difficulty in bending to tie her shoes and put on her tights: this physical restriction of movement was associated with mild pain in the lower back. Two years before admission she developed double vision, the images being vertically split, irrespective of the direction of gaze. This symptom lasted several months and required correction with prisms. It spon- taneously improved although it recurred, particularly when the patient was tired.

Investigations elsewhere showed positive acetylcholine receptor antibodies, weakly positive antinuclear antibodies (titres not known) and massively elevated interleukin 1 beta level of over 2000 units (upper limit of normal 20 units), on two occasions. An edrophonium test was negative and single-fibre EMG failed to demonstrate any neuromuscular junction abnormality. A CT of the head was normal as was a full length myelogram. An EEG demonstrated transient bioccipital slow wave activity.

Over the next nine months she had painless, mild dysphagia and a strangled quality to her voice. Admission to this hospital was precipitated by the occurrence of paroxysms of intense, painful muscle spasm, synchronously in all four limbs, associated with a choking sensation and profuse sweating. These episodes lasted for several hours and required parenteral administration of diazepam.

Past medical history included hypothyroidism diagnosed in 1983, for which she was taking thyroxine replacement, and a hysterectomy in 1984. She did not smoke and drank less than ten units of alcohol per week. There was no family history of neurological illness.

On examination she was mildly depressed but cognition was normal. Her face was flushed and she was sweating profusely. There was a mild spastic dysarthria. Examination of the spine showed globally reduced movement from the cervical to the lumbar regions with the shoulders fixed in elevation. There was a marked thoracic kyphoscoliosis and lumbar lordosis with profound paraspinal muscle spasm and rigidity. Her gait was normal, other than a movement "en bloc" of the trunk on turning. Saccadic eye movements were slow and hypometric in all directions, particularly to the right. Pursuit eye movements were limited upwards and to the right but improved with Dolls' Head manoeuvre. No nystagmus was observed. There was wasting of both temporalis muscles, a brisk jaw jerk and mild weakness of neck flexion. The rest of the cranial nerves were normal. Tone was normal in the upper limbs and no fasciculations were seen. There was symmetrical wast- 
ing of the shoulder girdle muscles and $4+/ 5$ weakness of the deltoids and triceps. The abdominal muscles were rigid. Tone in the lower limbs was normal and there was mild generalised wasting. Again, no fasciculations were seen. There was a $4+/ 5$ weakness of the iliopsoas and hamstrings bilaterally. She was areflexic and the plantar responses were both flexor. Sensory examination was normal. The patient demonstrated striking stimulus-sensitive myoclonus; when eliciting the jaw jerk or gently tapping the face in the "snout" area, brief, painful shock-like spasms were provoked synchronously in all four limbs.

General medical examination was normal with a resting pulse of 80 and BP 130/80.

Investigations included normal full blood count, urea and electrolytes, liver function tests, creatine kinase, blood glucose, chest radiograph and serum vitamin B12. Serological tests for syphilis were negative. She had an elevated free T4 $(32.5 \mathrm{pmol} / \mathrm{l}$; normal range 8.8-25.7 pmol/l) and a reduced TSH $(0.16 \mathrm{mU} / 1 ;$ normal range $0.4-3.5 \mathrm{mU} / \mathrm{l})$ and her thyroxine replacement was therefore reduced. An auto-antibody screen revealed a positive gastric parietal auto-antibody, positive thyroid thyroglobulin and thyroid microsomal auto-antibodies (titres 1/80 and $1 / 400$ respectively) and weakly positive antinuclear and acetylcholine receptor autoantibodies. A contrast enhanced CT scan of the head showed only mild cerebellar atrophy. MRI of the head and spine (axial STIR 2000/ 116 sequences for the head and sagittal $440 / 20$ and $1500 / 80$ for cervical and thoracic spine) was normal. The CSF was clear and colourless, with less than one white blood cell per microlitre, a protein of $0.28 \mathrm{~g} / 1$ and a glucose $3.0 \mathrm{mmol} / 1$ (blood glucose $5.4 \mathrm{mmol} / \mathrm{l}$ ). Isoelectric focusing of CSF proteins demonstrated oligoclonal IgG banding, unmatched with serum IgG. An EEG and upper limb somatosensory evoked potentials were normal. Extensive electrophysiological investigation confirmed the presence of continuous muscle fibre activity in the paraspinal muscles and brainstem reticular reflex myoclonus.

Testing for anti-GAD antibodies was performed using techniques previously described. ${ }^{2}$ In brief, with serum and CSF from the patient as a source of primary antibodies to immunostain sections of rat and human brain tissue, then using a light-microscopy immunocytochemistry technique (immunofluorescence and immunoperoxidase staining), both body fluids produced an intense immunostaining of all regions of the grey matter. By performing double-immunofluorescence experiments on rat brain using sheep antiserum to rat brain glutamic acid decarboxylase (GAD antiserum) the staining pattern produced by the patient's serum and CSF and the GAD antiserum were identical. It was thus concluded that the patient's serum and CSF were positive for anti-GAD antibodies. The specificity of this test for antiGAD antibodies has previously been shown to be over $98 \% .^{2}$ Since her initial admission in September 1989 the patient's disabling spasms and rigidity have slowly worsened but have responded partially to increasing doses of diazepam (current dose $35 \mathrm{mg}$ daily). Immunosuppressive therapy has not been used.

\section{Discussion}

The cervical emphasis of the rigidity, progression over three years to involve the bulbar musculature and lower spine, with loss of tendon jerks, nuclear and supranuclear gaze palsies and reticular reflex myoclonus all pointed towards the diagnosis of PEWR in this patient. It is thus possible to distinguish clinically PEWR from other causes of muscular stiffness and rigidity, particularly the stiff man syndrome and the syndrome of continuous muscle fibre activity. ${ }^{3-5}$

CT scanning showed a mild degree of cerebellar atrophy. More pronounced cerebellar atrophy has previously been reported in a similar case with a slowly progressive course over nine years. ${ }^{3}$ The MRI scan was, however, entirely normal and this contrasts with a recent report of PEWR in a 52 year old female in whom MRI demonstrated a lesion of the cervical cord and lower brainstem. ${ }^{6}$

Neuropathologically, PEWR is characterised by perivascular lymphocytic cuffing, increased microglial activity, astrocytic gliosis and neuronal loss, which mainly affects the brain stem and spinal cord, especially in the cervical region. ${ }^{47}$ Previously, this had further enabled a distinction to be made from other related disorders, particularly the stiff man syndrome, because in the few cases of stiff man syndrome evaluated at necropsy, no pathological findings in the nervous system had been found. ${ }^{8}$ However, a recent report of two cases of stiff man syndrome and autonomic instability examined neuropathologically has demonstrated perivascular gliosis in the spinal cord of one patient, and lymphocytic perivascular infiltration, predominantly in the spinal cord, brainstem and basal ganglia in the other. ${ }^{9}$

Recently, antibodies to gamma-aminobutyric acid containing (GABA-ergic) neurons, and in particular anti-GAD antibodies, have been described in the serum and CSF of 20 patients with stiff man syndrome, ${ }^{2}$ using light microscopy immunocytochemistry and Western blot analysis. There was a striking association with organ-specific autoimmune disease, particularly insulindependent diabetes mellitus, but also hypothyroidism, Graves' disease, vitiligo and possibly epilepsy. Impaired function of the inhibitory, GABA-ergic neurons has been implicated in the pathogenesis of stiff man syndrome. It was suggested that autoantibodies directed against GAD, the enzyme that synthesises GABA, could lead to reduced GABA-mediated central inhibition and thus alpha-motor neuron over-activity.

Our case had thyroid microsomal, thyroglobulin, acetylcholine receptor, gastric parietal and antinuclear auto-antibodies present in her serum. She was clinically and biochemically hypothyroid. The significance of 
the very raised interleukin 1 beta levels (over 2000 units) is uncertain. Levels as high as 150 to 200 units are seen in autoimmune diseases, particularly those with a vasculitic component (Behan PO, 1990- personal communication).

Our patient also had anti-GAD antibodies detected in her serum and CSF. The CSF IgG pattern was oligoclonal. It is possible that such antibodies are an epiphenomenon but it is tempting to postulate that in this woman the PEWR has an autoimmune basis, similar to that described for stiff man syndrome. If this were so, further questions would be raised regarding the pathogenesis of PEWR. For instance, since GAD is currently thought to be an intracellular enzyme,${ }^{10}$ what is the primary process initiating the disease? Furthermore, if there are pathogenetic and pathological similarities to stiff man syndrome, why should the clinical presentations differ? Do these conditions represent a clinical spectrum, as has previously been suggested? ${ }^{3}$ Future research may provide answers but will always be difficult because of the rarity of both conditions.

We are grateful to Dr Michele Solimena and co-workers of Milan University Medical School, Milan for performing the analysis on serum and CSF samples from our patient for antiGAD antibodies.

1 Howell DA, Lees AJ, Toghill PJ. Spinal internuncial neurones in progressive encephalomyelitis with rigidity. $J$ Neurol Neurosurg Psychiatry 1979;42:773-85.

2 Solimena M, Folli F, Aparisi R, Pozza G, De Camilli P. Autoantibodies to GABA-ergic neurons and pancreatic beta cells in stiff-man syndrome. $N$ Engl $J$ Med 1990;322:1555-60.

3 Leigh PN, Rothwell JC, Traub M, Marsden CD. A patient with reflex myoclonus and muscle rigidity: "jerking stiffman syndrome". J Neurol Neurosurg Psychiatry 1980;43:1125-31.

4 Whiteley AM, Swash M, Urich H. Progressive encephalomyelitis with rigidity: its relation to "subacute myoclonic spinal neuronitis" and to the "stiff man syndrome". Brain 1976;99:27-42.

5 Isaacs $H$. A syndrome of continuous muscle-fibre activity. $J$ Neurol Neurosurg Psychiatry 1961;24:319-25.

6 McCombe PA, Chalk JB, Searle JW, Tannenberg AEG, Smith JJ, Pender MP. Progressive encephalomyelitis with rigidity: a case report with magnetic resonance imaging findings. J Neurol Neurosurg Psychiatry 1989;52:1429-31.

7 Kasperek S, Zebrowski S. Stiff-man syndrome and encephalomyelitis. Arch Neurol 1971;24:22-30.

8 Layzer RB. Stiff-man syndrome-an autoimmune disease? $N$ Engl J Med 1988;318:1060-2.

9 Schwartzman MJ, Mitsumoto H, Chou SM, et al. Sudden death in stiff-man syndrome with autonomic instability. Ann Neurol 1989;26:166.

10 Kobayashi Y, Kaufman DL, Tobin AJ. Glutamic acid decarboxylase cDNA:nucleotide sequencing encoding an enzymatically active fusion protein. $J$ Neurosci 1987;9:2768-72. 